\title{
Hospital admission rates for COPD: the inverse care law is alive and well
}

\section{Rupert C M Jones}

In times of fiscal restrictions, health services need to invest resources where they will give the best return. Reducing hospital admissions by investing in improved disease management in the community is a prime target for those trying to save money. ${ }^{1}$ In their paper on the association of population and primary healthcare factors with hospital admissions for chronic obstructive pulmonary disease (COPD), Calderón-Larrañaga et al have shone an overdue spotlight on the determinants of admission rates. The authors have integrated routinely collected data sources on a breathtaking scale, using data from over 8000 practices caring for over 53 million people in England. They report a dramatic variation in admission rates from 125 to 646 per 100000 of population, which demands an explanation.

Among population factors, smoking rates and deprivation were unsurprisingly associated with higher prevalence and admission rates. The prevalence of undiagnosed COPD was also found to be an important contributor. To calculate undiagnosed prevalence it was necessary to estimate the expected prevalence rate using a mathematical model and to subtract the diagnosed prevalence determined by primary care disease registers. Several problems arise from this approach. Among others, disease registers are inaccurate-in one study $27 \%$ of those on COPD registers did not meet the spirometric criteria for COPD. ${ }^{3}$ Furthermore, the mathematical model was based on deprivation according to post codes, a process which may underestimate deprivation in practice populations. ${ }^{4}$

Various explanations can be postulated as to why the undiagnosed prevalence may affect admission rates. For instance, undiagnosed patients are likely to be denied interventions which might prevent admissions. On the other hand, a high undiagnosed prevalence rate may simply

Correspondence to Rupert C M Jones, Peninsula Medical School, Respiratory Research Unit, 1 Davey Road, University of Plymouth, Plymouth PL6 8BX, UK; rupert.jones@pms.ac.uk reflect a high disease burden due to factors such as smoking and deprivation; if so, the undiagnosed prevalence rate tells us nothing that is not already known.

Calderón-Larrañaga et al examined markers of the quantity and quality of care in primary care in relation to admission rates. Markers of quantity included the number of GPs and practice nurses per 1000 patients. Practice nurse supply was inversely related to admission rates, suggesting that employing more practice nurses may reduce admissions. Certainly the bulk of routine COPD care in the UK is performed by practice nurses. Griffiths et al found that COPD and asthma admissions were inversely related to nurse supply but the reverse applied for diabetes admissions, which suggests that the relationship between practice nurse supply and admissions may be complex and disease-specific. $^{5}$

One measure of quality is the ease of access to health practitioner appointments; this was assessed by CalderónLarrañaga et al using patient-reported data from every practice in England from the national GP survey. ${ }^{2}$ Noting the inverse relationship between admission rates and the ability to get an appointment within 2 days, it is tempting-yet premature-to conclude that patients with an exacerbation of COPD who are unable to get an appointment are more likely to be admitted. Other practice characteristics that are also likely to be important include: whether the practice prioritises COPD exacerbations as a reason for a same day appointment; whether they have telephone access to a health professional; and whether patients have home supplies of antibiotics and steroids with written action plans. Access to services outside the practice may also be relevant, such as access to community or hospital specialist respiratory nurses, acute exacerbation assessment services and pulmonary rehabilitation. There is a major variation in the provision of such services across the $\mathrm{UK}^{6}$ which may affect admission rates.

Calderón-Larrañaga et al also examined markers of the quality of clinical care for
COPD. Compared with GP access and quantity markers, quality markers were poor predictors of admission rates. The reported quality markers are blunt instruments for measuring complex services; only influenza vaccine uptake was significantly related to (reducing) admission rates. Undoubtedly there is concern about the variation in quality of services by practices in diagnosing $\mathrm{COPD}^{7}$ and appropriate use of drugs and referrals to rehabilitation, ${ }^{3}$ but these were not assessed. Whether early accurate diagnosis and guideline-based treatment in primary care, as advocated by the draft National Strategy for COPD, ${ }^{8}$ is associated with reduced admissions is not known.

Thus high COPD admission rates are found in deprived areas and rates are affected by the quality and quantity of primary care services. Reducing health inequalities has been and remains a political priority in the UK. ${ }^{1}$ The top $20 \%$ of primary care trusts with the poorest health outcomes (such as life expectancy) were designated as 'spearhead' primary care trusts and initiatives have been undertaken to improve the poor health of the population. Tools have been designed to map the problem and the underlying causes and to implement specific programmes to improve outcomes in diseases such as COPD. ' Unfortunately, mapping the problem is easier than solving it. Interventions to reduce the burden of COPD in deprived areas have looked at locally-driven integrated solutions including awareness-raising campaigns in the media targeted to encourage people at risk to be tested, educating primary care staff on the importance and benefits of COPD diagnosis and effective management. To reduce variance among practices, practices have been shown how their performance compares with others, encouraging them to 'pull up their socks'. Additionally, financial incentives have been instigated for improved services such as high quality spirometry.

While hard evidence is lacking, a combination of sticks and carrots does appear to have had some success in improving outcomes in COPD. Case studies such as the St Health primary care commissioning group have shown improved detection rates and the proportion of patients adjudged to be receiving guideline-based management has risen dramatically from $32 \%$ to $85 \%$. Admission rates have declined by $9.2 \%$ compared with a rise of $2.3 \%$ in adjacent practices. ${ }^{10}$ Clearly caution is needed when 
interpreting the changes in admission rates before and after an intervention as they are prone to bias. Despite the limitations, this sort of data will influence groups of GPs who will commission their own services in the brave new world of the latest NHS reforms. ${ }^{1}$

In deprived areas, optimal management of COPD may be affected by primary care service and patient factors. However, the practice performance cannot be disentangled from the characteristics of the population it serves. Surveys of patient experience are used as a marker of practice quality but depend on cooperation from patients. In areas of deprivation the results may be affected by many factors including literacy and language. Lack of patient engagement hampers the implementation of preventive health measures including early diagnosis by spirometry, smoking cessation, vaccination ${ }^{11}$ and pulmonary rehabilitation. ${ }^{12}$ Adherence to healthy lifestyles and to maintenance therapy is poor. $^{13}$

Primary care clinicians struggling to provide effective services for deprived populations therefore face extensive challenges including a high prevalence of COPD, many undiagnosed patients, problems in accessing and adhering to effective therapies and high costs from hospital admissions. Moreover, their patients have high risk factors such as smoking, poor nutrition and lack of exercise. These individuals are therefore prone to many long-term conditions including obesity, diabetes, ischaemic heart disease, mental illness, alcohol and substance misuse and represent a growing burden on the health service. ${ }^{14}$

As a result, deprivation in practice populations is associated with lower achievement of Quality and Outcomes Framework (QOF) scores, ${ }^{15} 16$ poorer clinical achievements in $\mathrm{QOF}^{17}$ and poorer results in the patient experience survey, thus reducing GP payments compared with national averages. As et al have demonstrated, deprived areas have fewer GPs, fewer practice nurses, a higher workload for COPD but with less financial reward than for GPs in affluent areas. ${ }^{10}$ Little change then from 1971 when Tudor Hart stated that the availability of good medical care tends to vary inversely with the need for the population served.

On a global scale these English experiments may appear minor. COPD is certainly the unsung harbinger of death in deprived populations in Africa where the interaction of smoking, deprivation, tuberculosis and HIV is unleashing a major epidemic of lung disease. ${ }^{18}$ In Africa, more people are expected to die from chronic respiratory disease than HIV by $2025 .{ }^{19}$ The message from the UK to the global health community is that, in order to address the rising challenge of COPD in deprived areas, resources need to be redistributed on the basis of need.

\section{Competing interests None.}

\section{Patient consent Obtained.}

Provenance and peer review Commissioned; not externally peer reviewed.

Published Online First 30 December 2010

Thorax 2011;66:185-186.

doi:10.1136/thx.2010.153569

\section{REFERENCES}

1. Department of Health. Equity and Excellence: Liberating the NHS. London: Department of Health, 2010.

2. Calderón-Larrañaga A, Carney L, Soljak $M$, et al. Association of population and primary healthcare factors with hospital admission rates for COPD in England: national cross-sectional study. Thorax 2011:66:191-6

3. Jones RC, Dickson-Spillmann M, Mather MJ, et al. Accuracy of diagnostic registers and management of chronic obstructive pulmonary disease: the Devon primary care audit. Respir Res 2008:9:62.

4. McLean G, Guthrie B, Watt G, et al. Practice postcode versus patient population: a comparison of data sources in England and Scotland. Int $J$ Health Geogr 2008; 7:37.

5. Griffiths P, Murrells T, Dawoud D, et al. Hospital admissions for asthma, diabetes and COPD: is there an association with practice nurse staffing? A cross sectional study using routinely collected data. BMC Health Serv Res 2010;10:276.

6. Royal College of Physicians. Report of the National Chronic Obstructive Pulmonary Disease Audit 2008: Resources and Organisation of Care in Acute NHS Units across the UK. London: Royal College of Physicians, 2008.

7. White $\mathbf{P}$, Wong $W$, Fleming $T$, et al. Primary care spirometry: test quality and the feasibility and usefulness of specialist reporting. $\mathrm{Br} \mathrm{J}$ Gen Pract 2007; 57:701-5

8. Department of Health Respiratory Team. Consultation on a Strategy for Services for Chronic Obstructive Pulmonary Disease (COPD) in England. London: Department of Health, 2010.

9. London Health Observatory. Health Inequalities Intervention Toolkit. London: London Health Observatory, 2010

10. Gupta A, Church S, Lacey S. The early detection of chronic obstructive pulmonary disease. Thorax 2010;65(Suppl IV):A174.

11. Coupland C, Harcourt S, Vinogradova $\mathrm{Y}$, et al. Inequalities in uptake of influenza vaccine by deprivation and risk group: time trends analysis. Vaccine 2007:25:7363-71.

12. Young $\mathbf{P}$, Dewse $M$, Fergusson $W$, et al. Respiratory rehabilitation in chronic obstructive pulmonary disease: predictors of nonadherence. Eur Respir $J$ 1999;13:855-9

13. Wamala S, Merlo J, Bostrom G, et al. Socioeconomic disadvantage and primary nonadherence with medication in Sweden. Int J Qual Health Care 2007:19:134-40.

14. Tian Y, Jager E, Stephen C, et al. Prevalence Study on Long Term Conditions in Birmingham. Birmingham: Birmingham Public Health Information Team, 2009. http://www.bhwp.nhs.uk/Files/Content/L/200/JSNA \%20LTCs\%20Final\%20Version.pdf.

15. Wright J, Martin D, Cockings S, et al. Overall quality of outcomes framework scores lower in practices in deprived areas. Br J Gen Pract 2006;56 277-9.

16. Ashworth M, Armstrong D. The relationship between general practice characteristics and quality of care: a national survey of quality indicators used in the UK Quality and Outcomes Framework, 2004-5. BMC Fam Pract 2006;7:68.

17. Saxena S, Car J, Eldred D, et al. Practice size, caseload, deprivation and quality of care of patients with coronary heart disease, hypertension and stroke in primary care: national cross-sectional study. BMC Health Serv Res 2007:7:96.

18. van Zyl Smit RN, Pai M, Yew WW, et al. Global lung health: the colliding epidemics of tuberculosis, tobacco smoking, HIV and COPD. Eur Respir J 2010;35:27-33.

19. World Health Organization. World Health Statistics. Geneva: World Health Organization, 2008. 